\title{
EVALUATING THE PERFORMANCE OF THE LOCATION- AIDED ROUTING-1P ROUTE DISCOVERY ALGORITHM
}

\author{
Ali Maqousi and Hussein Al-Bahadili \\ Department of Computer Science, Faculty of Information Technology, University of Petra, Amman, Jordan
}

Received 2013-07-07; Revised 2013-10-04; Accepted 2013-11-28

\begin{abstract}
Dynamic Routing Protocols (DRPs) are widely-used for routing information among mobile nodes in Mobile Ad Hoc Network (MANET) and establish and maintain connectivity within the network. A DRP comprises two main phases: route discovery and route maintenance. The route discovery phase involves transmission of large number of redundant control packets consuming significant portion of the nodes power and increase communication overheads. Recently, a new efficient and effective route discovery algorithm has been developed, namely, the LAR-1P algorithm, which combines two well-known routing protocols; these are: The Location-Aided Routing scheme 1 (LAR-1) and probabilistic algorithms. This study evaluates and compared the performance of the LAR$1 \mathrm{P}$ algorithm against the performance of a number of route discovery algorithms through simulation. For each simulation, the number of retransmissions and reachability are estimated and compared. The simulations results demonstrated that LAR-1P provides better performance than all other algorithms it is compared with, as it significantly reduces communication overheads while maintaining almost the same network connectivity.
\end{abstract}

Keywords: MANET, Route Discovery, LAR-1P, LAR-1, Probabilistic Algorithm, Pure Flooding

\section{INTRODUCTION}

A MANET is a set of wireless mobile nodes which are able to communicate with each other without relying on any established infrastructures or centralized administration. Therefore, each node in the network must be able to operate as router and needs to be capable of discovering and maintaining routes to every other node in the network (Al-Bahadili, 2013; Loo et al., 2011). Data in MANETs is usually forwarded to other mobile nodes in the network through reliable and efficient DRPs, which are part of the network layer software (Sensarma and Majumder, 2013). The DRPs are responsible for finding and deciding which output route data should be transmitted on. Example of DRPs include the Dynamic Source Routing (DSR), Ad Hoc OnDemand Distance Vector (AODV), Zone Routing Protocol (ZRP). DRPs usually consists of two main phases; these are: Route discovery and route maintenance (Hnatyshin, 2013; Mani et al., 2013).
Route discovery is usually executed when a source node desires to send a packet to some destination and does not already have a valid route to that destination; in this case, the source initiates a route discovery process to locate the destination (Wang et al., 2009). During the route discovery phase, the source node broadcasts a Route Request (RREQ) packet to its neighbors, which then forward the request to their neighbors and so on until the expiration of the packet. During the forwarding process, the nodes record in their route tables the address of the node from which first copy of the broadcast packet is received. Once the RREQ reaches the destination, the destination node responds with a route reply (RREP) packet back to the source through the route from which it first received the RREQ. Otherwise, if the RREQ packet expired before reaching the destination, then the node at which it expires, sends a Route Error (RERR) packet back to the source to initiate a new route discovery process (Yassein et al., 2011).

Corresponding Author: Ali Maqousi, Department of Computer Science, Faculty of Information Technology, University of Petra, Amman, Jordan 
Pure flooding is the simplest and most reliable mechanism proposed in the literature for route discovery in MANETs (Yassein et al., 2011). In pure flooding, each node rebroadcasts the message to its neighbors upon receiving it for the first time, starting at the source. However, pure flooding is costly where it costs $N$ transmissions in a network of $\mathrm{N}$ reachable nodes. Thus, pure flooding results in serious redundancy, contention and collisions in the network; such a scenario is undesirable and it is oftenly called the Broadcast Storm Problem (BSP) (Azizi, 2013).

Various flooding optimization algorithms have been developed to alleviate the effects of BSP during route discovery in MANETs by reducing the number of redundant retransmissions without significantly affecting network reachability. Such as the LAR-1 algorithm (Hnatyshin, 2013; Husain et al., 2010; Deb et al., 2009) and probabilistic broadcast (Hadji-nejad et al., 2010; Nand and Sharma, 2012; Al-Bahadili, 2010a; AlBahadili and Kaabneh, 2010).

The performance of LAR-1 significantly suffers from the high number of redundant retransmissions in highdensity zones (networks) (Hnatyshin and Asenov, 2010). While dynamic probabilistic usually provides excellent performance in high-density zones subject to using proper retransmission probability $\left(p_{t}\right)$ adjustment mechanism (Nand and Sharma, 2012; Al-Bahadili, 2010a).

A new route discovery algorithm has been proposed in Al-Bahadili (2013). It combines two algorithms, namely, the LAR-1 and probabilistic algorithms and it is referred to as the LAR-1P algorithm. In this algorithm, when receiving a RREQ, a node within the request zone rebroadcasts the RREQ with dynamically adjusted $\mathrm{p}_{\mathrm{t}}$. LAR-1P combines the better of the two algorithms. In low-density zones; the nodes assigned $\mathrm{p}_{\mathrm{t}}$ equal or close to unity, so that the algorithm acts as LAR-1, while a satisfactory $\mathrm{p}_{\mathrm{t}}$ is determined assigned in high-density zones, which means the algorithm acts as probabilistic algorithm.

The simulation results in (Al-Bahadili, 2013) demonstrated that for a uniform random node distribution on specific network area and for a certain simulation setup, LAR-1P reduces the number of retransmissions with slight reduction in reachability. The reduction in reachability can be avoided by proper $\mathrm{p}_{t}$ adjustment at low-density zones. Furthermore, LAR-1P can provide better average performance if the route discovery processes is performed more in high-density zones than in low-density zones.

This study evaluates the performance of the LAR-1P algorithm using the MANET simulator (MANSim) (Al-
Bahadili, 2010b). First, the effect of nodes density (total number of nodes in the network divided by the network area) on the performance of LAR-1P is investigated and compared against other algorithms like pure flooding, dynamic probabilistic and LAR-1. Second, for a certain nodes density $\left(4 \times 10^{-4}\right.$ node $\left./ \mathrm{m}^{2}\right)$, the effects of zones density on the performance of the LAR-1 and LAR-1P algorithms are investigated for one particular source node (Node 1).

The paper is organized as follows. Section 1 presents in addition to the above introduction, a review of the most recent and related work, description of the LAR-1, probabilistic broadcast and LAR-1P algorithms and the computational procedures adopted in this work. The materials and methods used in this study are described in Section 2. The simulation results and discussions are presented in Sections 3 and 4. Finally, Section 5 pointsout a number of conclusions and recommendations for future work.

\subsection{Literature Review}

A review of some of the most recent and related work to LAR-1P, LAR-1 and probabilistic algorithms is presented. In particular, this review summarizes the development steps of these algorithms. A fixed retransmission probability LAR-1P algorithm proposed, first, by Al-Bahadili et al. (2007). Later on, Al-Bahadili (2013) developed a new version of LAR-1P with dynamic probability, which will be described later. The effect of node density on the performance of the LAR-1P algorithm was investigated by Al-Bahadili et al. (2012).

Location information initially used by Ko and Vaidya (2000) to develop two different LAR schemes, LAR-1 and LAR-2 to reduce flooding communication overhead in ad hoc networks. Li et al. (2000) presented a modified version of LAR protocol, which was called LAKER (location aided knowledge extraction routing) protocol for MANETs. Boleng and Camp (2004) combined location information and nodes mobility feedback and pattern to create more efficient routing protocol that is capable of reducing routing communication overheads.

Deb et al. (2009) proposed a new location-aided routing protocol for GPS scarce MANET called the location-aided cluster based energy-efficient routing (LACBER). Another cluster-based LAR protocol for large scale MANETs was developed by Wang et al. (2009). Husain et al. (2010) presented a study on using LAR protocols for vehicular ad hoc networks (VANETs) in highway scenario. 
Hnatyshin and Asenov (2010) and Hnatyshin (2013) provided a study summarizing the use of geographical location information to reduce the control traffic overhead associated with the route discovery process of the AODV routing protocol. They introduced a new Geographical AODV (GeoAODV) protocol that relies on location information to reduce the flooding area to a portion of the network that is likely contains a path to destination. Furthermore, they compared GeoAODV performance with that of the LAR protocol and examined four mechanisms for reducing the size of the flooding area: LAR zone, LAR distance, GeoAODV static and GeoAODV rotate. They employed OPNET Modeler version 16.0 software to implement these mechanisms and to compare their performance through simulation.

Nand and Sharma (2012) proposed a probabilitybased broadcasting technique for routing protocol to trim down flooding problem. It uses node's remaining energy strength and threshold random delay to generate rebroadcast probability dynamically for the efficient broadcasting in route discovery. This technique was analyzed over reactive AODV protocol. The performance of modified protocol is analyzed over broadcast packets sent and end-to-end delay using NS2 simulator. Simulation results were observed and found that redundant transmission were reduced by 19 to 28 percent and hence improve network performance.

Probabilistic algorithm was first used by Haas $e t$ al. (2006) for route discovery in ad hoc networks and they called it a gossip-based (GOSSIP1) protocol. They used a predefined $\mathrm{p}_{\mathrm{t}}$ to decide whether or not a node forwards the control packets. Later on, Haas et al. (2006) developed a modified protocol, in which they gossip with $p_{t}=1$ for the first $h$ hops before continuing to gossip with $\mathrm{p}_{\mathrm{t}}<1$. Their results showed that they can save up to $35 \%$ message communication overheads compared to simple flooding in various network environments.

Tseng et al. (2002) examined the performance of probabilistic flooding for various network densities. They presented results for three network parameters, namely, reachability, saved rebroadcast and average latency, as a function of $\mathrm{p}_{\mathrm{t}}$ and network density. Sasson et al. (2003) developed probabilistic algorithm in which nodes would dynamically adjust their $\mathrm{p}_{\mathrm{t}}$ based on local topology information. Kim et al. (2004) introduced a probabilistic scheme in which a node dynamically adjusts its $p_{t}$ according to its additional coverage area. The additional coverage is estimated by the distance from the sender.

Scott and Yasinsac (2004) presented a dynamic probabilistic solution that is appropriate to solving BSPs in dense mobile networks. Barrett et al. (2005) introduced a probabilistic routing protocol for sensor networks, in which a sensor decides to forward a message with $p_{t}$ that depends on various parameters, such as the distance of the sensor to the destination, the distance of the source sensor to the destination, or the number of hops a packet has already travelled.

Viswanath and Obraczka (2005) developed an analytical model to study the performance of plain and probabilistic flooding in terms of its reliability and reachability in delivering packets showing that probabilistic flooding can provide similar reliability and reachability guarantees as plain flooding at a lower overhead. A probabilistic scheme that dynamically adjusts $\mathrm{p}_{\mathrm{t}}$ as per node distribution and node movement was proposed in (Zhang and Agrawal, 2005). The scheme combines between probabilistic and counter-based approaches.

Abdulai et al. (2006) studied the performance of the AODV protocol over a range of $\mathrm{p}_{\mathrm{t}}$. They focused on the route discovery part of the routing algorithm; they modified the AODV implementation to incorporate $\mathrm{p}_{\mathrm{t}}$. Simulations showed that setting efficient $\mathrm{p}_{t}$ has a significant effect on the performance of the protocol. The results also revealed that the optimal $p_{t}$ for efficient performance is affected by the prevailing network conditions such as traffic load, nodes density and mobility. Abdulai et al. (2007) also proposed two probabilistic methods for on-demand route discovery, that is simple to implement and can significantly reduce the overhead involved in the dissemination of RREQs. The two methods are: The adjusted probabilistic (AP) and Enhanced AP (EAP).

Yassein and Ould-Khaoua (2007) proposed a dynamic probabilistic algorithm to improve network reachability and saved rebroadcast. It determines $p_{t}$ by considering nodes density and speed. Abdulai et al. (2006) combined probabilistic and knowledge based approaches on the AODV protocol to enhance the performance of existing protocol by reducing the communication overhead incurred during the route discovery process. Hanash et al. (2009) proposed a dynamic probabilistic approach that can reduce broadcast redundancy in MANETs. Al-Bahadili (2010a) developed a new $\mathrm{p}_{\mathrm{t}}$ adjusting model, in which the neighbourhood densities are divided into three regions of different density ranges (low, medium and high). The performance of the new model was compared against pure and probabilistic algorithms.

Hadji-nejad et al. (2010) introduced a routing scheme for MANETs, which works well under a wide range of network topologies, nodes-density, coverage area size 
and nodes-mobility. The scheme is based on a novel enhancement of the hint-based probabilistic protocol. Instead of broadcasting extensive control packets for network topology information retrieval as that of conventional routing schemes, the scheme carefully reuses the feedback information carried in unicast packets for this purpose without overhead. The scheme is evalauted through both mathematical analysis and an extensive simulations study.

Nand and Sharma (2012) developed a probabilitybased broadcasting technique for routing protocol to trim down flooding problem. It uses node's remaining energy strength and threshold random delay to generate rebroadcast probability dynamically for the efficient broadcasting in route discovery. This technique was analyzed over reactive AODV protocol. The performance of modified protocol is analyzed over broadcast packets sent and end-to-end delay using NS2 simulator. Simulation results were observed and found that redundant transmission were reduced by 19 to 28 percent and hence improve network performance.

\subsection{The LAR-1 Algorithm}

The LAR-1 algorithm is based on two important concepts, namely, the Expected Zone (EZ) and the Request Zone (RZ). For EZ, consider a source node $S$ that needs to find a route to a destination node $D$ assuming $S$ knows the location of $D$ at time $t_{0}$ and the current time is $t_{1}$. Then, EZ of D, from the viewpoint of $S$ at time $t_{1}$, is the region that expected by $S$ to contain $D$ at time $t_{1}$. $S$ can determine EZ based on the knowledge that $\mathrm{D}$ was at location $\mathrm{L}$ at time $\mathrm{t}_{0}$ and travels with speed

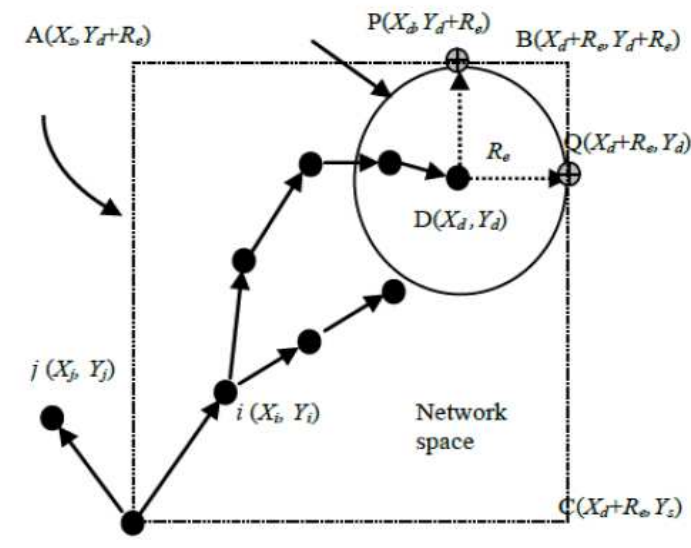

(a) Source node (S) outside the expected zone (EZ). $\mathrm{u}$, then $\mathrm{S}$ may assume that EZ is circular region of radius $\mathrm{R}_{\mathrm{e}}=\mathrm{u}\left(\mathrm{t}_{1}-\mathrm{t}_{0}\right)$, as illustrated in Fig. 1a (Husain et al., 2010).

If the actual speed happens to be larger than the average, then the destination may actually be outside EZ at time $t_{1}$ and vice versa. Thus, EZ is only an estimate made by $\mathrm{S}$ to determine a region that potentially contains $D$ at time $t_{1}$. If $S$ does not know a previous location of $D$, then $\mathrm{S}$ cannot reasonably determine EZ, in this case, the entire region that may potentially be occupied by the MANET is assumed to be EZ and the algorithm reduces to the basic flooding algorithm. In general, having more information regarding mobility of $\mathrm{D}$ can result in more appropriate EZ.

For RZ, again, consider $\mathrm{S}$ needs to determine a route to D. LAR-1 uses pure flooding with one modification. S defines (implicitly or explicitly) a RZ for the RREQ that contains both $\mathrm{S}$ and $\mathrm{D}$ and the locations of $\mathrm{S}$ and $\mathrm{D}$ as its opposite corners. A node forwards a RREQ only if it belongs to RZ (unlike pure flooding). To increase the probability that RREQ will reach $\mathrm{D}, \mathrm{RZ}$ should include EZ (Fig. 1a). Additionally, RZ may also include other regions around RZ (Al-Bahadili et al., 2012). Note that the probabilities of finding a path (in the first attempt) can be increased by increasing the size of RZ. However, route discovery overhead also increases with increasing size of RZ. Thus, there exists a trade-off between latency of route determination and the message overhead.

Now, let us move to discuss LAR-1 scheme. It uses an RZ that is rectangular in shape (Fig. 1).

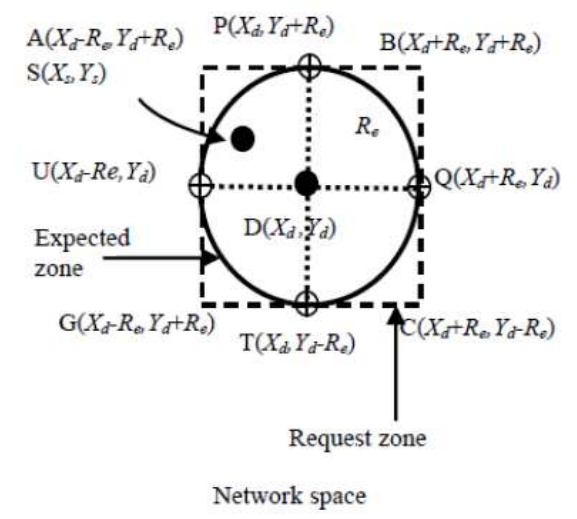

(b) Source node (S) within the expected zone (EZ).

Fig. 1. The LAR-1 algorithm 
It is assumed that $\mathrm{S}$ knows that $\mathrm{D}$ at location $\left(\mathrm{X}_{\mathrm{d}}, \mathrm{Y}_{\mathrm{d}}\right)$ at $t_{0}$. At $t_{1}, S$ initiates a new route discovery for $D$. It is also assumed that $\mathrm{S}$ knows the speed $\mathrm{u}$ with which $\mathrm{D}$ can move. Using this, $\mathrm{S}$ defines $\mathrm{EZ}$ at $\mathrm{t}_{1}$ to be the circle of radius $\mathrm{R}_{\mathrm{e}}=\mathrm{u}\left(\mathrm{t}_{1}-\mathrm{t}_{0}\right)$ centred at location $\left(\mathrm{X}_{\mathrm{d}}, \mathrm{Y}_{\mathrm{d}}\right)$. As stated before, instead of the average speed, $u$ may be chosen to be the maximum speed or some other function of the speed distribution. RZ is defined to be the smallest rectangle that includes current location of $S$ and $E Z$, such that the sides of the rectangle are parallel to the $\mathrm{X}$ and $\mathrm{Y}$ axes. In Fig. 1a, RZ is the rectangle whose corners are $S$, $\mathrm{A}, \mathrm{B}$ and $\mathrm{C}$, whereas in Fig. 1b, the rectangle has corners at point A, B, C and G. In Fig. 1, current location of $S$ is denoted as $\left(\mathrm{X}_{\mathrm{s}}, \mathrm{Y}_{\mathrm{s}}\right)$ and consequently $\mathrm{S}$ can determine the four corners of RZ.

$\mathrm{S}$ includes their coordinates with the initial RREQ when initiating route discovery. When a node receives a RREQ, it discards the request if it is not within the rectangle specified by the four corners included in the RREQ. For instance, in Fig. 1a, if i receives the RREQ from another node, $\mathrm{i}$ forwards the request to its neighbours, because $\mathrm{i}$ determines that it is within the rectangular $R Z$. When $\mathrm{j}$ receives the RREQ, it discards the request, as it is not within $\mathrm{RZ}$.

When $D$ receives the RREQ packet, it replies by sending a RREP packet. In case of LAR-1, D includes its current location and current time in the RREP packet. When $S$ receives this RREP packet (ending its route discovery), it records the location of $\mathrm{D}$. $\mathrm{S}$ can use this information to determine RZ for a future route discovery. It is also possible for D to include its current speed or average speed with the RREP packet. This information can be used in a future route discovery. Further details on LAR schemes can be found in (Hnatyshin, 2013; Deb et al., 2009; Wang et al., 2009).

\subsection{The Probabilistic Algorithm}

Probabilistic algorithm aims at reducing number of retransmissions, in an attempt to minimize the effects of the BSP in MANETs (Nand and Sharma, 2012). In this scheme, when receiving a RREQ, a node retransmits it with probability $\mathrm{p}_{\mathrm{t}}$ and with probability $1-\mathrm{p}_{\mathrm{t}}$ it discards the RREQ. A node is allowed to retransmit the same RREQ only once, i.e., if a node receives a RREQ, it checks to see if it has retransmitted it before, if so then it just discards it, otherwise it probabilistically retransmits it. Nodes identify the RREQ through its sequence number. The source node $\mathrm{p}_{\mathrm{t}}$ is always set to 1 , to ensure the source initializing a new RREQ.

The $\mathrm{p}_{\mathrm{t}}$ for intermediate nodes (all nodes except the source and the destination) is determined using either static or dynamic approach. In a static approach, a predetermined $\mathrm{p}_{\mathrm{t}}\left(0 \leq \mathrm{p}_{\mathrm{t}} \leq 1\right)$ is assigned for each node in the networks. While, in a dynamic approach, each node in the network locally determines it's $p_{t}$ depending on the number of first-hop neighboring nodes using certain probability distribution function; usually, $\mathrm{p}_{\mathrm{t}}=\mathrm{f}(\mathrm{k})$, where $\mathrm{k}$ is the number of first-hop neighbors.

In this study, the distribution function in Al-Bahadili (2010a) is used. This is because it demonstrated an excellent performance in comparison with other distribution functions under various network environments and configurations.

\subsection{The LAR-1P Algorithm}

The LAR-1P algorithm is straightforward as it is simply, when receiving a broadcast message, a node within the RZ rebroadcasts the message with dynamically calculated $p_{t}$ and each node is allowed to rebroadcast the received message only once. This reduces the number of retransmissions and also the node average duplicate reception, subsequently, the number of collisions and contentions at the receiver. The reduction in the number of retransmission will of course depend on the assigned $\mathrm{p}_{\mathrm{t}}$ and the algorithm is reduced to LAR-1 if the nodes' $\mathrm{p}_{\mathrm{t}}$ is set as unity.

It is important to recognize that reduction in the number of retransmission may introduce some reduction in network reachability. This is because if the number of intermediate nodes within RZ is small (low-density zones) and some of them will not rebroadcast the RREQ, which may result in a failure of delivery of the RREQ to destination, so that the source has to reinitiate a new RREQ.

It is expected that if the number of intermediate nodes within RZ is large (high-density zones), there will be insignificant reduction in reachability. Figure 2 outlines the procedure of the LAR-1P algorithm.

There are two values for the first-hop neighbors $(\mathrm{k})$ that can be considered for calculating $\mathrm{p}_{t}$ in dynamic probability LAR-1P:

- The first represents the total number of first-hop neighbors regardless of whether they are inside or outside RZ (same as in probabilistic algorithm)

- The second considers nodes inside RZ only, which is usually equal to or less than the first value

Normally, every node in the network updates the lists (records) of its first-hop neighbors at the start of each time interval, which is usually lasted for what is referred to as the pause time $(\tau)$. 


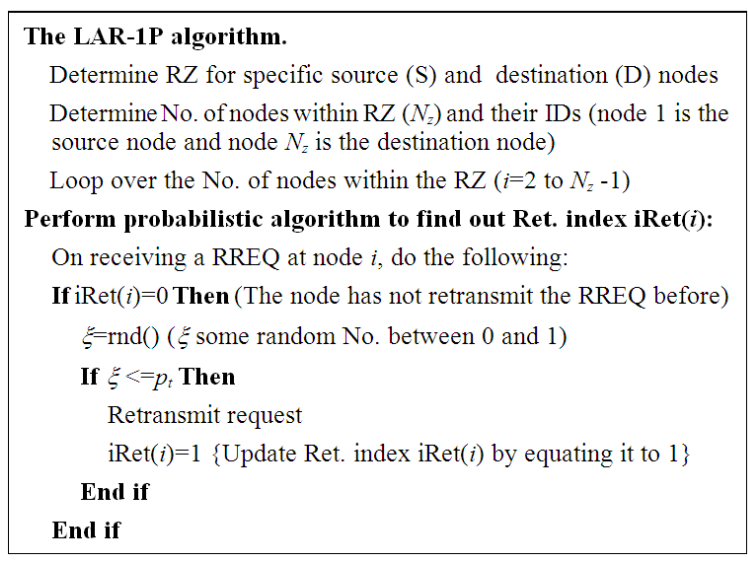

Fig. 2. The LAR-1P algorithm

It can be seen from the above description of LAR-1P that at high-density zones, the nodes will be given low $\mathrm{p}_{\mathrm{t}} \mathrm{s}$ inflicting a reduction in the number of retransmissions that depends on the assigned $\mathrm{p}_{\mathrm{t}} \mathrm{s}$. Which means the probabilistic algorithm is effective. While at low-density zones, the nodes will be allowed to retransmit the RREQ packet with high $\mathrm{p}_{\mathrm{t}}$, if it is unity or close to unity, then LAR-1P acts as LAR-1.

\subsection{Computational Procedures}

There are two computational procedures that have been implemented in MANSim (Al-Bahadili, 2010b). The first computational procedure computes the average parameters for a pre-specified S-D pair. In this procedure, the source initiates Q RREQs each time interval. The computation is repeated for a number of time intervals $(\mathrm{M})$, for each time interval, the nodes in the network are allowed to change their positions in the network. Therefore, the computed parameters should be averaged over $\mathrm{Q}$ and $\mathrm{M}$. The computed average values represent the average parameters associated with this particular S-D pair, but they may not reflect the average behavior of other S-D pairs. However, it has been found that for a network that has no probabilistic behavior, i.e., $\mathrm{p}_{\mathrm{t}}=1, \mathrm{Q}$ has no effect on the computed parameters and $\mathrm{Q}$ can be set to 1 .

The second computational procedure computes the average network parameters that can be considered as the average parameters for any S-D pair. This is calculated by looping over all nodes in the network as source nodes and each source tries to establish a route to all other nodes in the network as destination nodes. Furthermore, for each S-D pair, the source initiates Q RREQs as described in the first computation procedure.

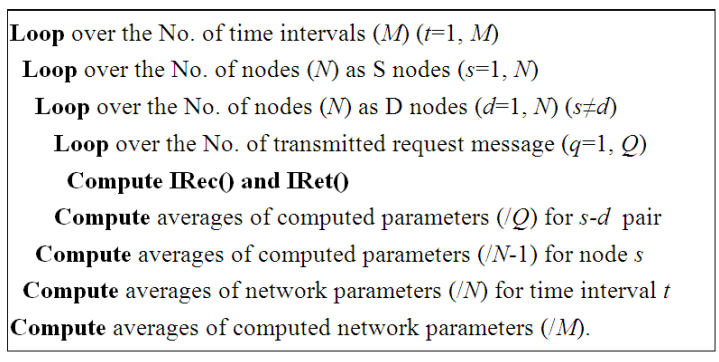

Fig. 3. Second computational procedure in MANSim

The computed parameters for each source are averaged over $\mathrm{Q}(\mathrm{N}-1)$ and then the average values are averaged over $\mathrm{N}$. In other words, the computed parameters are averaged over $(\mathrm{Q} \cdot \mathrm{N}(\mathrm{N}-1))$.

In order to consider nodes mobility, the above calculations are repeated, in an outer loop of size $\mathrm{M}$ and the computed parameters are averaged over M. In this case, the computed parameters may well represent the average behavior of any of the nodes in the network. For non-mobile (fixed) nodes, $M$ has no effect on the computed parameters and can be set to 1 . Figure 3 outlines the second computation procedure.

\section{MATERIALS AND METHODS}

The LAR-1P is simulated using MANSim network simulator, which is especially develped for evaluating the performance of various types of route discovery algorithms in MANETs (Al-Bahadili, 2010b). The performance of the algorithms is evaluated in terms of two well-known parameters (Al-Bahadili, 2013):

- Number of Retransmissions (RET), which is defined as the average number of retransmissions normalized to the total number of nodes in the network $(\mathrm{N})$

- Reachability (RCH), which is defined as the average number of reachable nodes by any node in the network normalized to $\mathrm{N}$; or the probability by which a RREQ packet will be successfully delivered from source to destination

In order to investigate the effect of nodes density $\left(\mathrm{N}_{\mathrm{d}}=\right.$ N/A) on the performance of LAR-1P, a number of simulations were performed using MANSim. The network environment and simulation setup can be described as follows: Each simulation starts by generating $\mathrm{N}$ nodes randomly distributed across a network area (A) of $500 \times 500 \mathrm{~m}$. All nodes are assumed to be transmitting with the same radio transmission Range (R) of $100 \mathrm{~m}$ and move with an average soeed (u) of $5 \mathrm{~m} \mathrm{sec}^{-1}$. 
One important parameter that needs to be carefully specified to obtain a more realistic network performance is the pause time $(\tau)$, which is defined as a period of time in which all nodes in the network are assumed motionless. In MANSim, $\tau$ is calculated as: $\tau=0.75 \cdot \mathrm{R} / \mathrm{u}$. Each simulation is carried-out for a certain period of time called simulation time $\left(\mathrm{T}_{\text {sim }}\right)$, which is divided into a number of intervals that are called mobility loops (M), each loop is of a period $\tau$. The input data for the simulations are given in Table $\mathbf{1}$.

In order to compare the performance of LAR-1P with other route discovery algorithms, we use MANSim to compute RET and $\mathrm{RCH}$ for three well-known route discovery protcols; these are: Pure flooding, dynamic probabilistic and LAR-1 (LAR-1P with $\left.p_{t}=1\right)$ uing the same network parameters and simulation data. Let us now remind ourselves with two important issues:

- The main requirement of any route discovery algorithm is to reduce RET without sacrificing $\mathrm{RCH}$

- $\mathrm{p}_{\mathrm{t}}$ in LAR-1P is calculated considering the first-hop neighbors positioned inside the RZ only

For each nodes density, the percentage reduction in $\mathrm{RCH}$ and RET are calculated by Equation 1 and 2:

Table 1. Simulation input data

\begin{tabular}{ll}
\hline Parameters & Value \\
\hline Geometrical model & Random node distribution \\
Network area $(A)$ & $500 \times 500 \mathrm{~m}$ \\
Number of nodes $(N)$ & $50,75,100,125,150$ nodes \\
Transmission radius $(R)$ & $100 \mathrm{~m}$ \\
Average node speed $(u)$ & $5 \mathrm{~m} / \mathrm{sec}$ \\
Simulation time $\left(T_{\text {sim }}\right)$ & $1800 \mathrm{sec}$ \\
Pause time $(\tau)$ & $15 \mathrm{sec}$ \\
Mobility loop size $(M)$ & 120 \\
\hline
\end{tabular}

$$
\begin{aligned}
& \mathrm{R}_{\mathrm{RCH}}=\frac{\mathrm{RCH}_{\mathrm{LAR}-1}-\mathrm{RCH}_{\mathrm{LAR}-1 \mathrm{P}}}{\mathrm{RCH}_{\mathrm{LAR}-1}} \\
& \mathrm{R}_{\mathrm{RET}}=\frac{\mathrm{RET}_{\mathrm{LAR}-1}-\mathrm{RET}_{\mathrm{LAR}-1 \mathrm{P}}}{\mathrm{RET}_{\mathrm{LAR}-1}} \times 100
\end{aligned}
$$

\section{RESULTS}

The results obtained for RET and $\mathrm{RCH}$ by the various algorihms (pure flooding, dynamic probabilistic, LAR-1 and LAR-1P) are listed in Table 2 and also plotted in Fig. 4 and 5. Table 3 presents the average $p_{t}\left(p_{t, a v g}\right)$ and the average $\mathrm{k}\left(\mathrm{k}_{\mathrm{avg}}\right)$ for the dynamic probabilistic and LAR- $1 \mathrm{P}$ algorithms.

In order to demonstrate the advantage of LAR-1P, let us consider the following example. Assume node 1 is the source and all other nodes are examined as destinations one at a time. For each S-D pair we count the number of nodes within the RZ $\left(\mathrm{N}_{\mathrm{z}}\right)$ including the source and the destination. Then, we count the number of cases in which $N_{z}$ lies within the ranges $(2-10,11-20,21-30, \ldots$,$) .$ For each range, we estimate $\mathrm{RCH}$ and RET for both LAR-1 and LAR-1P algorithms and the results obtained are summarized in Table 4.

\section{DISCUSSION}

Table 2 shows that the performance of LAR-1P varies in the same pattern as other algorithms. Also, it is found that in comparison with LAR-1, $\mathrm{R}_{\mathrm{RCH}}$ fluctuated around a very small percent with increasing nodes density, while $\mathrm{R}_{\mathrm{RET}}$ is increasing, which means more reduction in number of collisions and contentions.

Table 2. RET and RCH Comparison

\begin{tabular}{lllllrr}
\hline $\mathrm{N}$ & $\mathrm{N}_{\mathrm{d}}(\mathrm{N} / \mathrm{A})$ & Pure flooding & Dynamic probability & LAR-1 & LAR-1P & $(\%)$ \\
\hline RET & & & & & & \\
50 & $2 \times 10^{-4}$ & 0.715 & 0.652 & 0.087 & 0.084 & 3.45 \\
75 & $3 \times 10^{-4}$ & 0.932 & 0.771 & 0.129 & 0.108 & 16.28 \\
100 & $4 \times 10^{-4}$ & 0.975 & 0.718 & 0.147 & 0.115 & 21.77 \\
125 & $5 \times 10^{-4}$ & 0.983 & 0.643 & 0.159 & 0.115 & 27.67 \\
150 & $6 \times 10^{-4}$ & 0.987 & 0.589 & 0.161 & 0.109 & 32.30 \\
RCH & & & & & & \\
50 & $2 \times 10^{-4}$ & 0.767 & 0.750 & 0.396 & 0.394 & 0.51 \\
75 & $3 \times 10^{-4}$ & 0.961 & 0.946 & 0.772 & 0.612 & 4.52 \\
100 & $4 \times 10^{-4}$ & 0.995 & 0.981 & 0.857 & 0.720 & 6.74 \\
125 & $5 \times 10^{-4}$ & 0.999 & 0.990 & 0.894 & 0.859 & 5.13 \\
150 & $6 \times 10^{-4}$ & 1.000 & 0.995 & & 3.91 \\
\hline
\end{tabular}


Ali Maqousi and Hussein Al-Bahadili / Journal of Computer Science 10 (3): 423-433, 2014

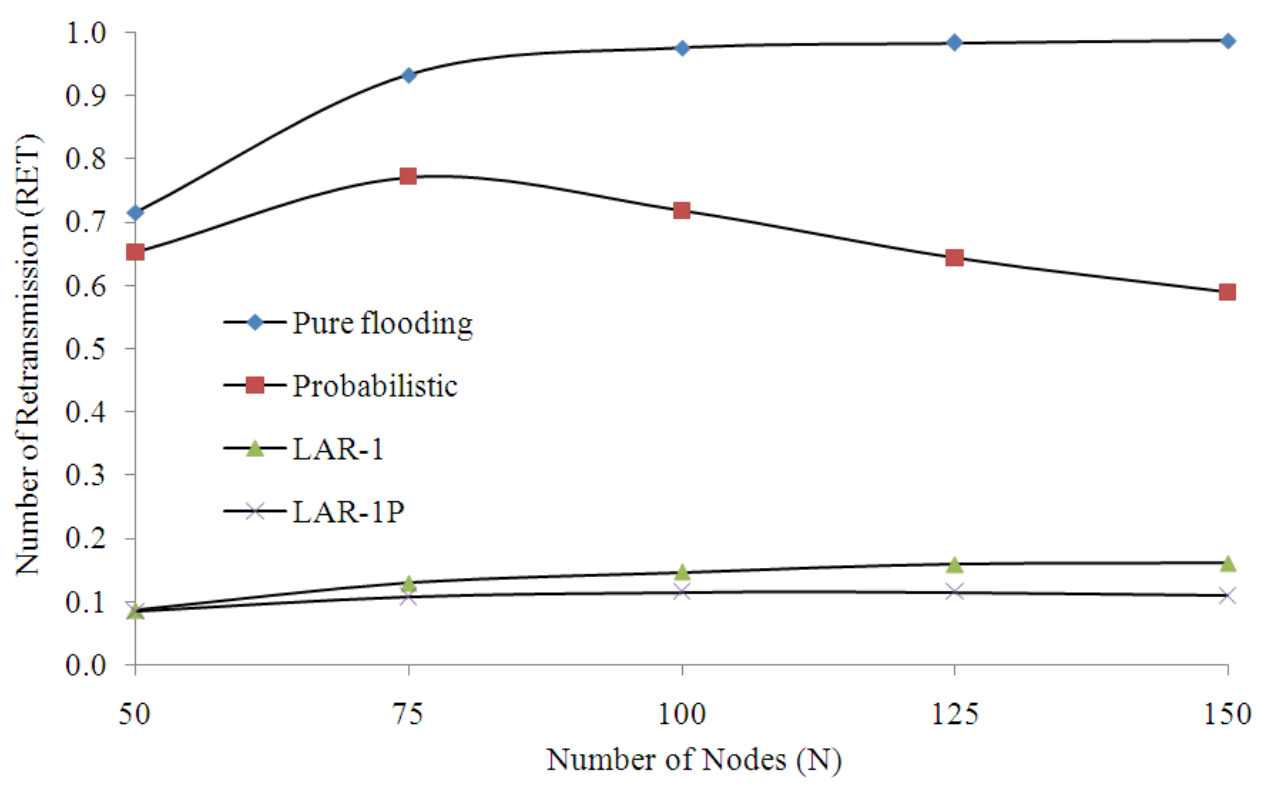

Fig. 4. RET comparison

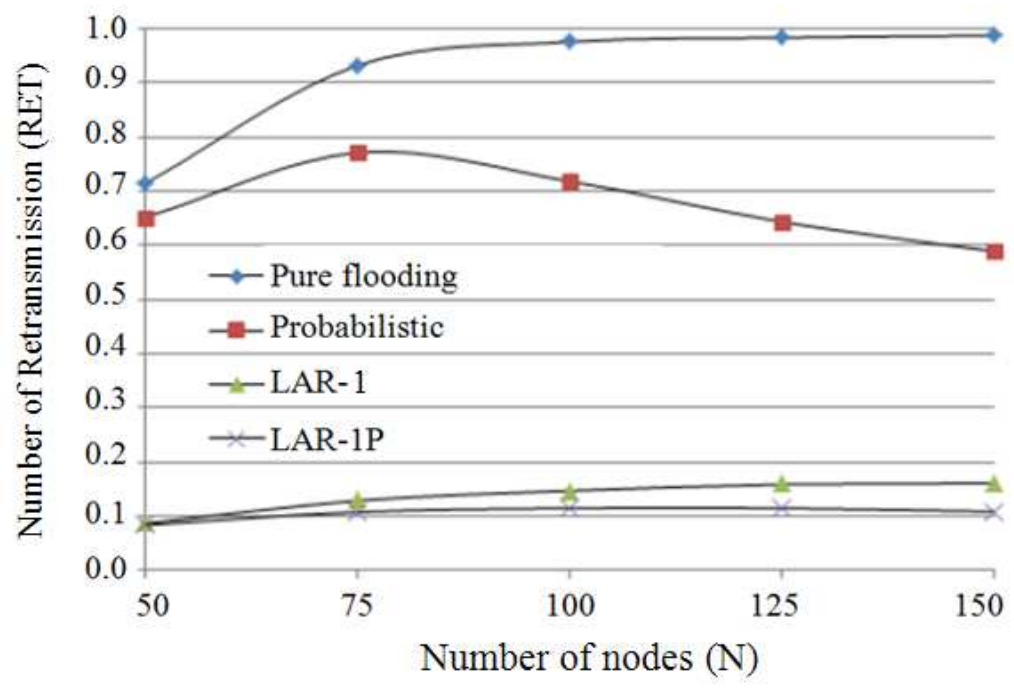

Fig. 5. RCH comparison

Table 3. Comparing $\mathrm{p}_{\mathrm{tavg}}$ and $\mathrm{k}_{\text {avg }}$

\begin{tabular}{|c|c|c|c|c|c|}
\hline \multirow[b]{2}{*}{$\mathrm{N}$} & \multirow[b]{2}{*}{$\mathrm{N}_{\mathrm{d}}(\mathrm{N} / \mathrm{A})$} & \multicolumn{2}{|l|}{$\mathrm{p}_{\mathrm{t}, \text { avg }}$} & \multicolumn{2}{|l|}{$\mathrm{k}_{\mathrm{avg}}$} \\
\hline & & Dynamic probability & LAR-1P & Dynamic probability & LAR- 1P \\
\hline$\overline{50}$ & $2 \times 10^{-4}$ & 0.935 & 0.981 & 4.913 & 3.418 \\
\hline 75 & $3 \times 10^{-4}$ & 0.844 & 0.946 & 7.262 & 4.945 \\
\hline 100 & $4 \times 10^{-4}$ & 0.749 & 0.904 & 9.562 & 6.247 \\
\hline 125 & $5 \times 10^{-4}$ & 0.661 & 0.848 & 12.092 & 7.865 \\
\hline 150 & $6 \times 10^{-4}$ & 0.601 & 0.796 & 14.373 & 9.401 \\
\hline
\end{tabular}


Table 4. Comparison between the LAR-1 and LAR-1P

\begin{tabular}{llllllll}
\hline & & \multicolumn{2}{l}{ LAR-1 } & & \multicolumn{2}{l}{ LAR-1P } \\
$N_{z}$ & $Z$ & RET & RCH & RCH & RET & RCH & RCH \\
\hline $2-10$ & 40 & 0.048 & 32 & 0.800 & 0.043 & 31 & 0.775 \\
$11-20$ & 27 & 0.127 & 22 & 0.815 & 0.113 & 21 & 0.778 \\
$21-30$ & 11 & 0.222 & 10 & 0.909 & 0.186 & 10 & 0.909 \\
$31-40$ & 11 & 0.328 & 11 & 1.000 & 0.263 & 11 & 1.000 \\
$41-50$ & 10 & 0.431 & 10 & 1.000 & 0.310 & 10 & 1.000 \\
\hline
\end{tabular}

Table 3 shows $k_{\text {avg }}$ is increasing with increasing nodes density. For each node density, $\mathrm{k}_{\mathrm{avg}}$ for dynamic probabilistic is greater than that for LAR-1P. This is because we only consider first-hop neighbors that are positioned inside RZ. As a result of that $\mathrm{p}_{\mathrm{t} \text {,avg }}$ is decreasing with increasing nodes density for the two algorithms and for the same node density, $\mathrm{p}_{\mathrm{t} \text {,avg }}$ for dynamic probabilistic is less than LAR-1P. Next, to analyze the performance of the algorithms for a single node density value of $4 \times 10^{-4}$ nodes $/ \mathrm{m}^{2}$. Pure flooding provides the highest $\mathrm{RCH}$ of $99.5 \%$ at $97.5 \%$ of the nodes in the network engaged in RREQ retransmission. The probabilistic algorithm achieves a very high $\mathrm{RCH}$ of $98.1 \%$ but only reduces RET by $\sim 28$ to $72 \%$.

LAR-1 reduces RET to a very low level of $14.7 \%$ at the cost of reducing $\mathrm{RCH}$ to $77.2 \%$. In other words, LAR-1 introduces a significant reduction of $\sim 85 \%$ in RET but at the same time reducing $\mathrm{RCH}$ by $\sim 22 \%$. These results represent an average behavior over uniform distribution of zones densities. But if most of route discovery processes involve searching the route in highdensity zones, then RET will be definitely higher than $14.7 \%$. For example, for RZs that confine all nodes, $\mathrm{RCH}$ and RET will be the same as or close to that in pure flooding. Furthermore, in high-density zones the delay is much higher than in low-density zones.

It can be seen in Table 2 that for LAR-1P, RCH is $5.2 \%$ less than LAR-1 and the associated RET is also less by $3.2 \%$. However, when we look at zone scale, for example, for high-density zones, LAR-1P attains the performance of dynamic probabilistic of $98.1 \%$ reachability at $\sim 30 \%$ reduction in RET. Thus, if most route discovery processes involve high-density zones, a reduction in RET is always equal to or close to $30 \%$. On the other hand, for low-density zones, the estimated $\mathrm{p}_{\mathrm{t}} \mathrm{s}$ are always close to 1, i.e., inflicting pure flooding within RZ, which means LAR-1 is the acting algorithm.

Now, let us explain the results in Table 4. $Z$ in the second column gives the number of RZs that contains a number of nodes within the range indicated in the first column, i.e., the number of RZs that contains number of nodes within the range from 2 to 10 is 40 and 27 contains a number of nodes between 11 to 20 .

For LAR-1, consider the case of low-density zone (210 ), in 32 out of the 40 , the source and destination were able to establish a connection and 8 of them were not, giving $\mathrm{RCH}$ of $80 \%$. Due to the small number of nodes within RZs, RET was only $4.8 \%$. The LAR-1P provides slightly less RCH and RCH. For high-density zone (4150), LAR-1P accomplishes the same RCH of 10 out of 10 at a significant reduction in RET from $43.1 \%$ for the LAR-1 to $31 \%$. In high-density zone, reduces RET significantly and almost maintains the same $\mathrm{RCH}$.

\section{CONCLUSION}

In this study, we present performance evaluation of the of the LAR-1P route discovery algorithm in MANETs. The algorithm is especially developed to enhance the performance of the LAR-1 alforithm as it combines two widely-used algorithms, namely, LAR-1 and probabilistic algorithms. The obtained results showed that the performance of the LAR-1 algorithm can be enhanced when combined with the probabilistic approach especially in high density zones, where we can achieve more reduction in the number of retransmissions against insignificant reduction in network reachability. Moreover, the LAR-1P algorithm provides the performance as LAR-1 in low-density zones.

For future work, we highly recommend to investigate other network parameters, such the node speed, mobility model, pause time, radio transmission range.

\section{REFERENCES}

Abdulai, J., M. Ould-Khaoua and L.M. Mackenzie, 2007. Improving probabilistic route discovery in mobile ad hoc networks. Proceedings of the 32nd IEEE Conference on Local Computer Networks, Oct. 15-18, IEEE Xplore Press, Dublin, pp: 739746. DOI: 10.1109/LCN.2007.114 
Abdulai, J., M. Ould-Khaoua, L. Mackenzie and M. Bani-Yassin, 2006. On the forwarding probability for on-demand probabilistic route discovery in MANETs. Proceedings of the 22nd Annual UK Performance Engineering Workshop (UKPEW'06), Poole, Dorset, UK, pp: 9-15.

Al-Bahadili, H. and K. Kaabneh, 2010. Analyzing the performance of probabilistic algorithm in noisy MANETs. Int. J. Wireless Mobile Networks, 2: 8395.

Al-Bahadili, H., 2010a. Enhancing the performance of adjusted probabilistic broadcast in MANETs. Mediterranean J. Comput. Networks, 6: 138-144.

Al-Bahadili, H., 2010b. On the Use of Discrete-Event Simulation in Computer Networks Analysis and Design. In: Handbook of Research on Discrete Event Simulation Environments: Technologies and Applications, Abu-Taieh, E.M.O. and A.A.R. ElSheikh (Eds.), IGI Global Snippet, Hershey, PA., ISBN-10: 1605667757, pp: 414-442.

Al-Bahadili, H., 2013. A new route discovery algorithm in MANETs combining location-aided routing and probabilistic algorithms. Mediterranean J. Comput. Networks, 9: 48-55.

Al-Bahadili, H., A. Maqousi and R.S. Naoum, 2012. Analyzing the effect of node density on the performance of the LAR-1P Algorithm. Int. J. Inform. Technol. Web Eng., 7: 16-29. DOI: 10.4018/jitwe.2012040102

Al-Bahadili, H., O. Al-Basheer and A. Al-Thaher, 2007. A location aided routing-probabilistic algorithm for flooding optimization in MANETs. Proceedings of the Mosharaka International Conference on Communication, Networking and Information Technology, (MIC-CNIT '07), Amman-Jordan.

Azizi, R., 2013. Performance study and simulation of an anycast protocol for wireless mobile ad hoc networks. Int. J. Wireless Mobile Networks, 5: 1933. DOI: $10.5121 /$ ijwmn.2013.5302

Barrett, C.L., S.J. Eidenbenz, L. Kroc, M. Marathe and J.P. Smith, 2005. Parametric probabilistic routing in sensor networks. Mobile Networks Applic., 10: 529544. DOI: 10.1007/s11036-005-1565-X

Boleng, J. and T. Camp, 2004. Adaptive location aided mobile ad hoc network routing. Proceedings of the IEEE International Conference on Performance, Computing and Communication, (CC'04), IEEE Xplore Press, 423-432. DOI: 10.1109/PCCC.2004.1395048
Deb, D., S.B. Roy and N. Chaki, 2009. LACBER: A new location-aided routing protocol for GPS scarce MANET. Int. J. Wireless Mobile Networks, 1: 2235.

Haas, Z.J., J.Y. Halpern and L. Li, 2006. Gossip-based ad hoc routing. IEEE/ACM Trans. Network., 14: 479-491. DOI: 10.1109/TNET.2006.876186

Hadji-nejad, S., M. Rahbar and H. Mehrgan, 2010. Synergy between phenothiazines and oxacillin against clinical isolates of methicillin-resistant Staphylococcus aureus. Tropical J. Pharmaceutical Res., 9: 243-249.

Hanash, A.M., A. Siddique, I. Awan and M. Woodward, 2009. Performance evaluation of dynamic probabilistic broadcasting for flooding in mobile ad hoc networks. Simulat. Model. Practice Theory, 17: 364-375. DOI: 10.1016/j.simpat.2008.09.012

Hnatyshin, V. and H. Asenov, 2010. Design and implementation of an OPNET model for simulating GeoAODV MANET routing protocol. Proceedings of the OPNETWORK International Conference, Session: Wireless Ad Hoc and Wireless Personal Area Networks, (SWWPAN' 10).

Hnatyshin, V., 2013. Improving MANET routing protocols through the use of geographical information. Int. J. Wireless Mobile Networks, 5: 133. DOI: 10.5121/ijwmn.2013.5201

Husain, A., B. Kumar and A. Doegar, 2010. A study of Location Aided Routing (LAR) protocol for vehicular ad hoc networks in highway scenario. Int. J. Eng. Inform. Technol., 2: 118-124.

Kim, J.S., Q. Zhang and D.P. Agrawal, 2004. Probabilistic broadcasting based on coverage area and neighbor confirmation in mobile ad hoc networks. Proceedings of the IEEE Global Telecommunication Conference Workshops, Nov. 29-Dec. 3, IEEE Xplore Press, Dallas, pp: 96-101. DOI: 10.1109/GLOCOMW.2004.1417556

Ko, Y.B. and N.H. Vaidya, 2000. Location-Aided Routing (LAR) in mobile ad hoc networks. Wireless Networks, 6: 307-321. DOI: 10.1023/A:1019106118419

Li, J., J. Jannotti, D.S.J. De Couto, D.R. Karger and R. Morris, 2000. A scalable location service for geographic ad hoc routing. Proceedings of the 6th International Conferenceon Mobile Computing and Networking, Aug. 6-11, ACM Press, Boston, MA, USA., pp: 120-130. 
Loo, J., J.L. Mauri and J.H. Ortiz, 2011. Mobile ad hoc Networks: Current Status and Future Trends. 1st Edn., CRC Press Publisher, ISBN-10: 1439856508, pp: 538.

Mani, U., R. Chandrasekaran and V.R.S. Dhulipala, 2013. Study and analysis of routing protocols in mobile ad hoc network. J. Comput. Sci., 9: 15191525. DOI: $10.3844 /$ jessp.2013.1519. 1525

Nand, P. and S.C. Sharma, 2012. Probability based improved broadcasting for AODV routing protocol. Proc. Eng., 50: 222-231. DOI: 10.1016/j.proeng.2012.10.027

Sasson, Y., D. Cavin and A. Schiper, 2003. Probabilistic broadcast for flooding in wireless mobile ad hoc networks. Proceedings of the IEEE Wireless Communicatio and Networking Conference, Mar. 20-20, IEEE Xplore Press, New Orleans, LA, USA., pp: 1124-1130. DOI: 10.1109/WCNC.2003.1200529

Scott, D.J. and A. Yasinsac, 2004. Dynamic probabilistic retransmission in ad hoc networks. Proceeding of the International Conference on Wireless Networks, (ICWN' 04), pp: 158-164.

Sensarma, D. and K. Majumder, 2013. An efficient ant based QoS aware intelligent temporally ordered routing algorithm for MANETs. Int. J. Comput. Networks Commun., 5: 189-203. DOI: 10.5121/ijcnc. 2013.5415
Tseng, Y.C., S.Y. Ni, Y.S. Chen and J.P. Sheu, 2002. The broadcast storm problem in a mobile ad hoc network. J. Wireless Networks, 8: 153-167. DOI: 10.1023/A:1013763825347

Viswanath, K. and K. Obraczka, 2005. Modeling the performance of flooding in wireless multi-hop ad hoc networks. Comput. Commun., 29: 949-956. DOI: 10.1016/j.comcom.2005.06.015

Wang, Y., L. Dong, T. Liang, X. Yang, X. and D. Zhang, 2009. Cluster based location-aided routing protocol for large scale mobile ad hoc networks. IEICE Trans. Inform. Syst., 5: 1103-1124.

Yassein, M.B. and M. Ould-Khaoua, 2007. Applications of probabilistic flooding in MANETs. Int. J. Ubiquitous Comput. Commun., 1: 1-5.

Yassein, M.B., S.F. Nimer and A. Y. Al-Dubai, 2011. A new dynamic counter-based broadcasting scheme for mobile ad hoc networks. J. Simulat. Model. Practice Theory, 19: 553-563. DOI: 10.1016/j.simpat.2010.08.011

Zhang, Q. and D.P. Agrawal, 2005. Dynamic probabilistic broadcasting in MANETs. J. Parallel Distributed Comput., 65: 220-233. DOI: 10.1016/j.jpdc.2004.09.006 